\title{
Abstract: Physiological Parameter Estimation from Multispectral Images Unleashed
}

\author{
Sebastian J. Wirkert ${ }^{1}$, Anant S. Vemuri ${ }^{1}$, Hannes G. Kenngott ${ }^{2}$, \\ Sara Moccia ${ }^{1,3,4}$, Michael Götz ${ }^{2}$, Benjamin F. B. Mayer ${ }^{2}$, \\ Klaus H. Maier-Hein ${ }^{5}$, Daniel S. Elson ${ }^{6,7}$, Lena Maier-Hein ${ }^{1}$
}

1 Div. of Computer Assisted Medical Interventions, DKFZ, Heidelberg, Germany

2 Dept. for General, Visceral and Transplantation Surgery, Heidelberg University Hospital, Germany

3 Dept. of Advanced Robotics, Istituto Italiano di Tecnologia, Genoa, Italy

4 Dept. of Electronics, Information and Bioengineering, Politecnico di Milano, Italy

${ }^{5}$ Div. for Medical and Biological Informatics, DKFZ, Heidelberg, Germany

${ }^{6}$ Hamlyn Centre for Robotic Surgery, Imperial College London, UK

7 Dept. of Surgery and Cancer, Imperial College London, UK

$$
\text { s.wirkert@dkfz-heidelberg.de }
$$

Multispectral imaging in laparoscopy can provide tissue reflectance measurements for each point in the image at multiple wavelengths of light. These reflectances encode information on important physiological parameters not visible to the naked eye. Fast decoding of the data during surgery, however, remains challenging. While model-based methods suffer from inaccurate base assumptions, a major bottleneck related to competing machine learning-based solutions is the lack of labelled training data. In this paper, we address this issue with the first transfer learning-based method to physiological parameter estimation from multispectral images. It relies on a highly generic tissue model that aims to capture the full range of optical tissue parameters that can potentially be observed in vivo. Adaptation of the model to a specific clinical application based on unlabelled in vivo data is achieved using a new concept of domain adaptation that explicitly addresses the high variance often introduced by conventional covariance-shift correction methods. According to comprehensive in silico and in vivo experiments our approach enables accurate parameter estimation for various tissue types without the need for incorporating specific prior knowledge on optical properties and could thus pave the way for many exciting applications in multispectral laparoscopy.

This work was first presented at MICCAI 2017 [1].

\section{Literaturverzeichnis}

1. Wirkert SJ, Vemuri AS, Kenngott HG, et al. Physiological parameter estimation from multispectral images unleashed. In: Medical Image Computing and ComputerAssisted Intervention - MICCAI 2017. Lect Notes Comput Sci. Springer, Cham; 2017. p. 134-141. 